\title{
“LE DONNE SONO PESCATRICI DI VITE PERDUTE"1
}

Serena Scionti

\begin{abstract}
"Siamo poeti./Vogliateci bene da vivi di più /da morti di meno/che tanto non lo sapremo" 2 .

"Il talento femminile è un talento innato, una disposizione originaria, un assoluto virtuosismo nel conferire al finito un senso. La donna concilia l'uomo e se stessa col mondo, è in armonia con l'esistenza in una misura che l'uomo non conosce; poiché la donna spiega la finitezza, essa è la vita profonda dell'uomo: una vita tranquilla e nascosta, come è sempre la vita delle radici" ${ }^{3}$.
\end{abstract}

Quattro personalità dal "talento innato", quattro autrici contemporanee che "pescano vite perdute", quattro donne, di picche, di quadri, di fiori, di cuori: Natalia Veronesi Prada, medico che salva vite africane; Mariella Mischi, che richiama a vita Paul Celan; Alina Rizzi, che recupera amori smarriti; Gabriella Baracchi, che ritrova la sua infanzia. Sopra tutte, figura di spicco nel panorama nazionale della scrittura femminile, Vivian Lamarque, che fiori quadri cuori picche tutti assume, e che di vita ripesca la propria.

Natalia Veronesi Prada, donna di picche -simboleggianti il pensiero, la combattività, il dolore e le difficoltà; pediatra, con le sue due raccolte Giorni a piene mani e I grandi fiumi verdi e le colline, pubblicate fra il 2002 e il 2004 rispettivamente da Book e Ibis, ci conduce a leggere una lunga vita, divisa tra Nord e Sud, tra la Como familiare e il Burundi. La sua levità poetica ritrae scenari paradisiaci, profumati eucalipti e frangipane "dolcearoma", quasi danzanti sulla pagina dai versi semplici, insieme a vesti millecolori, nella seducente notte africana «segata dall'esile lima dei grilli». Ma con uguale efficacia di similitudini la Veronesi canta anche un'Africa dilaniata sotto i silenzi di «lucide costellazioni», in cui clamori bellici irrompono e pioggia mista a sangue dilava terreni spaccati. Con versi dai toni elegiaci che si chinano sulle distruzioni, lamenta «profughi braccati a morte», hutu e tutsi che si affrontano in una guerra in cui anche l'uomo bianco ha la sua parte; donna europea, autocriticamente afferma che «l'Europa distilla veleni/più dolci del fiore di loto:/il denaro cambia la vita». Provoca, la Veronesi, noi lettori di poesia, ad

\footnotetext{
${ }^{1}$ Il titolo "Le donne sono pescatrici di vite perdute" è tratto dal romanzo di DON DE LILLO, Underground, editore Scribner, New York, 1997.

${ }^{2}$ La quartina di Vivian Lamarque è posta ad esergo della sua opera omnia, Poesie, edita per Mondadori nel 2002.

${ }^{3}$ Le parole di Cesare Pavese sono reperibili in FRANCO VACCANEO, Cesare Pavese, Una biografia per immagini: la vita, i libri, le carte, i luoghi, Gribaudo, 2001.
} 
una "letteratura come vita", ad una poesia che con i valori umani si confronti: «Scoprirò l'uomo più vero/nei versi dei vostri poeti/o nei letti del mio ospedale?». Una vita vissuta nell'impegno, plenis manibus, «come se non dovesse mai finire», che solo nella maturità ha voluto tradursi in canto poetico, quasi a sottolineare la priorità dell'agire sociale rispetto alla contemplazione letteraria; una poesia che ha accompagnato in sordina una vita intera $-i$ primi testi furono scritti sin dagli anni Quaranta-, e solo tardivamente ha voluto farsi dono di parola per il pubblico; parola che è però seconda alla Parola, al Verbo di Dio. Nell'autunno delle tragedie Dio è Primavera, in una feconda alternanza stagionale che diventa metafora della vita umana e della poesia stessa: «Illusione, poesia,/ non ci lasciare: sei la primavera/a cui docile il cuore/rifiorisce». I versi della dottoressa Veronesi percorrono sessant'anni di stupore, di sogni, di veglie sulla sofferenza altrui e sulla propria inquietudine, rivelando uno sguardo partecipe all'umanità dolente, con cui la poetessa invoca un Dio presente ma silenzioso.

La presenza di Dio permea fortemente anche la poesia di Mariella Mischi, veneta. Laureata in psicologia clinica, ha svolto l'attività di psicoterapeuta, per poi consacrarsi totalmente al demone della scrittura. A partire dal 1997, ha pubblicato Meridiana d'amore, Avalon-la spina e la rosa, Karate dō-via della mano vuota, Seme della metamorfosi, Lettere d'amore di due poetesse (con Alda Merini), Canzoniere di Giulietta e Romeo, e infine, Alle porte dei ritorni, in cui predomina uno stile nominale, l'accumulatio di immagini, anche metaforiche, nella dominanza cromatica dell'azzurro, colore celestiale per eccellenza. Il divino è la cifra per capire la scrittura appassionata di Mariella Mischi: «Benedico la Sostanza carnale, eucaristia degli amanti». E del divino pare soffiare in ogni verso la ruah, che col respiro umano si fonde e confonde, tra paesaggi orientali, deserti, ed oasi. Donna di quadri, è inquietamente divisa tra accenti spirituali e un eros che il logos vuole dominare, la ragione ricondurre a ragione. Se nel Seme della metamorfosi la fisicità era espressa schiettamente, nel Canzoniere per Giulietta e Romeo è allusiva, velata, sospirata. Poesie d'amore, recita il sottotitolo di questo epistolario poetico con andamento amebeo, che riecheggia scenari bucolici di amori pastorali. L'eros spiritualizzato e filosoficamente teorizzato richiama il drammatico carteggio tra Abelardo e Eloisa, ma la sensualità ricalca il Cantico dei Cantici, l'inno erotico veterotestamentario di grande pienezza amorosa, mirabile sintesi di carnalità e spiritualità. Amore, Eros, Cupido è sempre personificato, ed incede con solennità da litanie sacre. Ma nella poetica della Mischi esso è compagno inscindibile di Thanatos. Amore e morte, memore Platone. «Viviamo per morire»-dicono gli amanti. Amore è nel contempo divinità greca e mistica agape cristiana; così l'amata, è Dea e Madonna, tra angeli e Sibille, Muse e preghiere. Grazie ad allitterazioni curate, assonanze, sapiente collocazione degli accenti tonici nel verso, l'eroe e l'eroina percorrono il loro fatale cammino invasati d'amore, consacrati da pura follia nell'alcova dell'urna. Cos'altro è l'amore se non una «pazzia molto discreta, un'amarezza che soffoca, una dolcezza che fa bene?». "Donna di intelligenza amorosa" -così la definisce l'amica Alda Merini- la Mischi gioca col dissidio ratiolinsania, acuito, tra l'altro, dalla dinamica dell'assenza/presenza. «Pazzi gli amanti nella loro febbre» combattono sulla pagina la drammatica pugna tra ratio e moira, costringendo il lettore ad una verticalizzazione ardita, ad un pindarico passaggio fra terra e cielo, fra carne e anima: «Breve è la sponda tra il corpo e lo spirito quando due amanti si uniscono». 
Amanti uniti oltre la morte, in eterno dialogo, come nell'ultima raccolta, Alle porte dei ritorni. In essa il poeta Paul Celan -Paul Antschel, poeta romeno di lingua tedesca suicidatosi nella Senna nel 1970- rivive con gli innamorati versi della Mischi, attraverso cento canti, in una discesa ad inferos ed una Resurrezione. Tutta la raccolta è all'insegna della morte, che subito nell' ingredior del poema è volta in amore. Sub specie mortis appaiono oggetti e persone, in visioni oniriche, nel ferace binomio di eros e tanatos. Amore e morte. Morta la madre Friederike di Celan, e il figlio François, morta la Mater della poetessa, mirabilmente cantata in versi franti e drammatici, elegiaci pure sono i vivi: ai figli Mariella Mischi dedica Elegia per Chiara ed Elegia per Mattia, quasi li presagisse perduti negli addii connaturati alla vita adulta. Il suicidio di Celan è ritratto con dantesco realismo, l' «arida carcassa» è condotta allo Stige tra limo e viluppi di alghe; ma gli accenti dell'Inferno si scolorano nel «fiume della Genesi», ove «la deriva luminosa attende». L'attesa di una pienezza amorosa è rimandata alla vita ultraterrena.

Terrena, invece, terrestre e concreta è Alina Rizzi, donna di cuori. Comasca, è una giovane poetessa che si è affermata con un romanzo erotico pubblicato da Borrelli nella collana Il pizzo nero ma che ha trovato i suoi accenti più freschi nel racconto e soprattutto nei versi. Oltre ad alcune esili plaquettes pubblicate da Pulcinoelefante, cioè Oriente, Hic et nunc e Ardere spargendo, si è fatta conoscere con le raccolte Rossofuoco, di Lietocolle, e Il frutto sillabato, per Dialogolibri. Leggendo i testi della Rizzi ogni donna può compiere un viaggio all'interno del proprio io affettivo femminile, acquisendo maggior consapevolezza rispetto alle passioni da cui è animata. Rossofuoco, minuscolo e attaccato -come guidogozzano- per un minimalismo del quotidiano, nel continuum poetico di versi senza punteggiatura, senza maiuscole, quasi indistinti se non fosse per i titoli, pure minuscoli, che ci immettono in un discorrere ininterrotto, rafforzato dall'uso insistito degli enjambements, attraverso sussurri, in un canto che non osa, nel colloquio intimo con un generico 'tu', cui la raccolta è dedicata. Questo 'tu' è il compagno della condivisa e quieta passione giornaliera, il complice di gesti d'amore vissuti fra i fornelli, mentre si sparecchia. Di là dal prevalente hic et nunc amoroso, uno sguardo pure si volge oltre l'effimero, in dinamiche di separazione e contemplazione: ci sono giorni in cui l'amore coglie l'attimo, e allora sono labbra, baci, carezze; giorni invece in cui il presente è già scaduto, e si prostrano nel vuoto, non sono mai davvero nostri. Allora il dono più grande che l'amata può fare all'amato, è il tempo: quello presente, della vita insieme; quello passato, del ricordo, quando il fuoco dello sguardo gli incendiò il cuore, secondo la casistica classico-medievale, e infine quello del futuro, che si invererà in frutto d'amore incarnato.

Il frutto sillabato, è il titolo della seconda raccolta, partorito da donna creativa-creatrice, frutto di viscere e d'intelletto, frutto di passione e di volitiva dedizione. Se frutto è figlio di fiore, Il frutto sillabato della poetessa Alina Rizzi è il raccolto di una maturazione poetica che già ha fiorito il pregevole Rossofuoco. Una plaquette rossa, per tessere il rosso filo del discorso amoroso attraverso testi che saporano di ferialità, intessuta di trasporti e di litigi, come l'hanno cantata Anne Sexton, Adrienne Rich, Alda Merini, i cui versi sono posti in esergo alle tre sezioni della silloge. 'Lei' scrive una lunga lettera d'amore a 'lui', fatta di componimenti brevi, un canto di sentimenti alterni, ora passionali ora stanchi, a giorni accesi, a giorni ribelli . 'Lui' le risponde invitandola a risillabare il frutto d'amore che pareva avvizzito, sfatto nella serialità di giornate sempre uguali, quando «un malore velenoso/dilagante tra le bocche unite/separa scrivanie confinanti/come isole di fronte» e 
«sguardi piccoli per colazione» rivelano che ci si prende a rate «temendo un furto/quasi convinti di restituirci un giorno/io persa tu più ricco». Ci si illude allora, di «barare fino al prossimo incontro», di riprendersi da «quello scoppio in mezzo al cuore/che cauti non convinti/abbiamo detto amore», con la speranza di una nuova fioritura. E' la vicenda di ogni amore, che racconta la poetica di Alina Rizzi, teso tra «imperiosi ritorni quotidiani» e «passioni pomeridiane/prima di cena un po' immusonite»; efficaci metafore per fissare l'iter di relazioni vissute nell'alternarsi delle stagioni. Una primavera allora si spera, dopo il muto letargo nivale, con la convinzione che panta rei e che di nuovo -ciclo umorale femminile«il desiderio metterà germogli come collane».

Germogli, e fiori tutti, in Gabriella Baracchi, donna di fiori. Comasca, a lungo insegnante, ha donato alla sua città tre libri, cimentandosi in diversi generi testuali nei quali ha dimostrato cura e maestria. Il primo lavoro col quale si è resa nota, nel 1993, è il romanzo Il vestito di sacco, impostosi anche a livello nazionale. La materia autobiografica di un'infanzia difficile si è travasata anche all'opera che ha seguito il fortunato romanzo, i racconti brevi di Con la faccia dei giorni storti, quasi poemetti in versi, prose poetiche. Nel 2004 il dettato della Baracchi si è rarefatto ulteriormente, e il racconto si è stilizzato in versi poetici. Componimenti brevissimi, con la saggezza e il fulmen in clausula degli epigrammi, o l'icasticità degli aforismi. Retrospezioni introspettive, mai oleografie paesaggistiche, piuttosto correlativi oggettivi, dove il fiore, il cielo o la rondine prefigurano stati d'animo, carichi di memoria pesante. Versi essenziali, sobri, perfettamente equilibrati, classici, che ci penetrano come profumo lieve ma durevole, spesso gonfi di presagi oscuri che costringono a sostare e a riflettere. Epifanie, improvvisi squarci di memorie d'anni addietro, lacrimae rerum dice Vincenzo Guarracino. Frammenti è il titolo dell'opera, quasi a suggerire che i pochi versi che spiccano sulla chiara pagina sono il continuum di una trama segreta, affiorano da un parlato che li preannuncia e precede, o da un mondo altro, e continuano poi il loro messaggio nella nostra interiorità, creandovi un'atmosfera ineludibile, che ci avvince. Rare le rime, e le assonanze, i versi sono liberi da schemi metrici; le similitudini si riferiscono sempre al mondo naturale («entri nella mia giornata/come pioggia in un prato» «sei tenero paziente come l'erba/che tagliata rispunta»). In ogni componimento l'elemento naturalistico è quasi un pretesto per inabissarsi nei meandri dell'animo, che in mezzo a madre natura si pone, ammirandone con estasi l'armonia e utopisticamente desiderandola. Versi quieti, contemplativi, in cui i sentimenti aspri sono espressi con la pacatezza di metafore tratte dal comportamento degli animali o degli agenti atmosferici: pioggia, vento, nebbie, cani. Su tutto si stende il silenzio. Anche il dolore, che incide la raccolta, non è mai urlato, bensì ossimorico grido silenzioso, trattenuto e scavato nell'interiorità: «Dal sonno mi svegliò un urlo/il mio/ma la bocca era chiusa». Se il cuore è in fiamme e in lacrime, tuttavia il volto è di pietra, i gesti si compiono e si ripetono lenti e misurati. Disillusione e senso di morte, affrontati e vinti solo grazie alla vivacità della natura, il cui eterno sfiorire e rifiorire invita a non disperare. Nell'immensità del cielo e del lago la corporeità individuale del singolo, ingombrante e mortale («mi porto in giro come una cosa/che non so dove posare»), pare stemperarsi e perdersi; docile, il corpo si trasumana in un volo di gabbiano o scompare dietro la collina con un'ala di poiana. Tuttavia nostalgia, stanze vuote, malinconia, pesantezza vitale, continuano a stridere con la leggerezza della natura. Ma la morte, sereno traguardo, ci farà eguali. 
Eguali, polvere nella terra dell'uguagliante humus ci farà infine la morte, centrale nel lungo percorso di Vivan Lamarque edito nell'antologia poetica Poesie, pubblicata da Mondadori nel 2002. Nota anche come scrittrice per bambini, Vivian Lamarque è poetessa per adulti, nonché giornalista e traduttrice. Donna di fiori, o meglio di pratoline, dall'apparente lectio facilis («è quasi facile fare una poesia/basta prendere un pezzetto/di carta e una matita, è come/per la terra fare un filo d'erba/una margherita»); donna di cuori, innamorata di madri, padri, amanti, figli; donna di quadri, alla ricerca di un tetragono $u b i$ consistam; donna di picche, tormentata dal dolore.

Vivian Daisy Donata Comba Provera Pellegrinelli Lamarque: molti nomi per il molto travagliato feuilleton -così lo definisce- della sua autobiografia, protagonista della prima raccolta, Teresino, che uscì nel 1981. Nella prima sezione, Conoscendo la madre, Vivian come Pollicino è abbandonata, ma trova genitori adottivi; tuttavia il trauma della separazione, dell'abbandono, non la lascerà per la vita intera, che la fisserà "bambina" nella scrittura e negli amori adulti, riflessi di un amore materno alle origini negato. Segue L'amore mio è buonissimo, fantasmatico refrain ricco di espressioni tipicamente infantili, e poi Il primo mio amore erano due, in cui si narra di due amati adolescenti gemelli, ma si allude alle due madri, la naturale e la adottiva, a conferma della fusione inconscia tra amore infantile e amori adulti. Donna-bambina, si fa piccolina, anche fisicamente, «mangia la neve», si percepisce Cenerentola esclusa dal convito d'amore, dal nido felice di una famiglia: il bisogno insaziabile di essere amata, apprezzata, lo diluisce tacitamente nella quotidianità delle minestre, e come una formicuzza ha bisogno di capitalizzare affetti per i tempi freddi: e non è mai sufficiente, a causa della sua regressione emotiva, del desiderio recondito di tornare al grembo materno. Come un personaggio delle fiabe, parla di sé in terza persona nella sezione Il tuo posto vuoto, nel tentativo forse di distaccarsi ed elaborare le proprie angosce, o come se fossero gli altri a bisbigliare di lei, di quanto sta male, poverina, per il nuovo lutto della separazione dal marito, in cui cercava padre e madre. L'unico calore umano che le resta certo è una stufetta accesa, persa lei dietro a gesti ossessivi, infantili, come quando i bimbi non devono schiacciare la riga delle mattonelle, se no chissà cosa accadrà. Ad arginare il senso di morte che aleggia, interviene la sezione $H o$ una bella bambina, cantata con versi dolci e piani, quasi di Saba, senza pretese. Confermano l'immagine modesta, e il dono che di sé vuole fare, le dichiarazioni di poetica in Poeti: ma come i bimbi Vivian è goffa, sbaglia regalo, compie azioni che antiteticamente risultano sgradite al compagno d'amore, sempre idealizzato. Quella della Lamarque si autodefinisce poesia illegittima, oppure poesia malata, rebus difficile, irrisolvibile, come la vita. Poesia malata, infreddolita, che chiede di morire, come il piangente crepuscolare Corazzini; poesia che vuole volare via, fare castelli in aria, sradicarsi da radici che, tanto, non ci sono; poesia svagata, trasognata, immaginifica, fiabesca: «Splendidissima era la vita accanto a lui sognata./Nel sogno tra tutte prediletta la chiamava./E nella realtà?/La realtà non c'era, era abdicata./Splendidissima regnava la vita immaginaria». Sogna di volare via anche il Teresino (Tesero, il paese dove Vivian nacque? Terezin, il lager dove morivano i bambini?) della sezione eponima, novello Pollicino, o forse uomo amato ridotto a bambino, che -come lei- si muove tra parole spezzate, frante tra un verso e l'altro, e rinnova la violenza delle stragi innocenti della storia.

Le tre raccolte pubblicate successivamente, Il signore d'oro, Il signore degli spaventati, Poesie dando del lei, sono dedicate tutte all'analista B. M., cui la poetessa si rivolse per 
affidargli il proprio tormento esistenziale e del quale ineluttabilmente si innamorò, come transfert vuole; in esse si accentua anche l'idealizzazione della figura maschile, che già fu nei confronti del padre adottivo, morto a soli 34 anni. Il "signore" d'oro è in trono, e a lui ci si deve rivolgere dicendo ossequiosamente "un signore", come fanno i bambini. L'inevitabile negazione della reciprocità amorosa è scandita dai molti "non" anaforici, divieti che segnano il distacco e la lontananza. La narrazione di quest'amore impossibile procede attraverso botta e risposta, affermazioni conquistate attraverso interrogative dirette. Come i mille perché dei bambini, i punti di domanda si susseguono e chiedono conferme. Ma la porta del nido del dottor B.M. resta chiusa, e il paraklausithyron è drammatica nenia. L' adorato analista junghiano è il signore degli "spaventati", cioè di tutti i suoi pazienti in analisi, «bambini persi/presi per essere trovati», come Hansel e Gretel. Spererebbe, lei donna bambina, di essere svegliata come la bella addormentata da un bacio del principe azzuro B.M.: non accadrà, e lo stato di torpore mortifero continuerà ad accompagnarla.

Nell'ultima raccolta, Una quieta polvere, del 1996, vengono ripresi i temi della storia personale di Teresino. Madri padri figli presenta il dato ossessivo della madre: «La morte è una Madre che abbandona» e Vivian è una bimba cattiva che forse è stata abbandonata per colpa sua: «Facevo la brava bambina per conquistarti mammina. Corteggiamento vano». La poetessa vede famiglia dappertutto, financo negli animali o negli oggetti antropomorfizzati, quasi animisticamente. Anni dopo arriverà a conoscere i due fratelli e la sorella, membri del nido spezzato che tuttavia non si ricomporrà, anzi si frammenterà ulteriormente con la morte della madre adottiva ottantenne e con la separazione da Paolo Lamarque. Sola compagna fedele, ad aeternum, la scrittura, incarnata nel fedele Pennino, che titola la seconda sezione. Umile, dice di sè «sono una poetina media/normale/da due righe e mezzo/sulla garzantina universale». Una originale poetessa dai molti neologismi, con uno stile piano e colloquiale ma incisivo, fatto di frequentissime iterazioni delle parole, come il "cammina cammina" delle fiabe, o i "millissimi" superlativi dei modi di dire del codice segreto dei bimbi («conterò fino a mille/poi non l'aspetterò più»). Un'interiore coazione a ripetere, una ridda di sentimenti compulsivi sono marcati sulla pagina da anafore, anadiplosi e chiasmi in discorsi diretti liberi, senza le virgolette. Con occhi e lessico di bambina pare sdrammatizzare anche la morte, («è venuto il babau e non s'è vista più»), grazie all'uso di frequenti ossimori («freddo tepore»), rime facili, e dell'imperfetto dei giochi di finzione ("Facciamo che io ero.."), che mischia oniricamente fatti reali a cose vagheggiate. La quasi assenza di punteggiatura e le similitudini semplici, l'uso dei proverbi, le parole in scriptio continua, soprattutto nel dittico sostantivo/aggettivo, la capacità di giocare con i versi («la mia settimana è un settenario / con gli accenti su martedì e venerdì / al sabato il tono cala / risale il lunedì) ne fanno infine una "poetina" da molte righe sulla Garzantina!

Cuore della raccolta, la sezione Questa quieta polvere, poemetto ispirato alla Dickinson in cui i versi autografi sono intervallati da 53 citazioni tratte dalle favole e da altri poeti cari, tra i quali appunto l'eletta Dickinson.

Dopo le sezioni Poesie dando del lei (altre) e Cercasi: poesie per un trasloco, in cui protagonista è la casa nido, con tutta la famiglia unita, nuove tematiche presenta la sezione Fine millennio, dove la psiche si decentra finalmente da sé per osservare gli ultimi della scala sociale, i nuovi cittadini di Milano: gli immigrati, gli emarginati "vu cumprà". 
Come i fiori infine, ci presenta una Lamarque quale femminea San Francesco, capace di curvarsi sulle creature più umili e con esse aspettare serena la conclusione della vita terrena: «Quando mi ricordo della morte guardo diversamente i fiori e l'erba/li accarezzo/preparo la nostra futura amicizia/saremo così vicini». La tranquilla accettazione di vita e morte prosegue nel lungo poemetto de L'albero, -l'albero uomo, cui abbandonarsi, alto, su cui annidarsi o da cui lasciarsi cullare- soffuso di echi pascoliani, compendio poetico e summa di una vita; così nelle Poesie dedicate, in cui uno sguardo benevolo sugli altri, pacificato, si prende infine la rivincita sugli abbandoni sempre patiti. Separata figlia e moglie separata, è finalmente unita come madre e come nonna di "rosellinaMicol". 